\title{
Robotica
}

http://journals.cambridge.org/ROB

Additional services for Robotica:

Email alerts: $\underline{\text { Click here }}$

Subscriptions: Click here

Commercial reprints: $\underline{\text { Click here }}$

Terms of use : $\underline{\text { Click here }}$

\section{Environment perception based on LIDAR sensors for real road applications}

\author{
F. García, F. Jiménez, J. E. Naranjo, J. G. Zato, F. Aparicio, J. M. Armingol and A. de la Escalera
}

Robotica / Volume 30 / Issue 02 / March 2012, pp 185 - 193

DOI: 10.1017/S0263574711000270, Published online: 24 May 2011

Link to this article: http://journals.cambridge.org/abstract S0263574711000270

How to cite this article:

F. García, F. Jiménez, J. E. Naranjo, J. G. Zato, F. Aparicio, J. M. Armingol and A. de la Escalera (2012). Environment perception based on LIDAR sensors for real road applications. Robotica, 30, pp 185-193 doi:10.1017/S0263574711000270

Request Permissions : $\underline{\text { Click here }}$ 


\title{
Environment perception based on LIDAR sensors for real road applications

\author{
F. García †*, F. Jiménezł, J. E. Naranjo§, J. G. Zato§, F. Aparicioł, \\ J. M. Armingol $\dagger$ and A. de la Escalera $\dagger$
}

\author{
$\dagger$ Universidad Carlos III de Madrid, Laboratorio de Sistemas Inteligentes Avda, De La Universidad 30, 28911 Leganés \\ (Madrid), Spain \\ $\ddagger$ Universidad Politécnica de Madrid, INSIA, Carretera de Valencia, km.7, 28031 Madrid, Spain \\ $\S$ Universidad Politécnica de Madrid, E.U. de Informática, Carretera de Valencia, km.7, 28031 Madrid, Spain
}

(Received in Final Form: March 10, 2011. First published online: May 24, 2011)

\section{SUMMARY}

The recent developments in applications that have been designed to increase road safety require reliable and trustworthy sensors. Keeping this in mind, the most up-todate research in the field of automotive technologies has shown that LIDARs are a very reliable sensor family. In this paper, a new approach to road obstacle classification is proposed and tested. Two different LIDAR sensors are compared by focusing on their main characteristics with respect to road applications. The viability of these sensors in real applications has been tested, where the results of this analysis are presented.

KEYWORDS: Data fusion; Intelligent vehicles; ADAS; LIDAR.

\section{Introduction}

Trustworthy sensors are key elements regarding current road safety applications. In recent years, advances in information technologies have lead to more intelligent and complex applications that are able to deal with a large variety of situations. These new applications are known as Advance Driver Assistant Systems (ADAS). One of the main features of ADAS is the detection of dangerous situations while warning the driver in advance. However, the lack of trustworthy sensors that can deal with and anticipate such situations makes this a tough task. In this context, LIDARs have proved to be a reliable source for information and are capable of handling the most demanding applications.

A new approach for obstacle detection and classification using LIDARs is presented; this application attempts to differentiate between obstacles that are commonly found during real road situations.

In this work, a comparative analysis of two different 2D LIDAR techniques has been carried out. Different tests have been performed, which have involved a high variety of movements and have been designed to verify the viability of these sensors for road safety applications.

\footnotetext{
* Corresponding author. E-mail: fegarcia@ing.uc3m.es
}

\section{State of the Art}

ADAS require trustworthy sensors, which provide reliable information on the surroundings. In this context, the most recent attempts, based on driver technologies, such as DARPA Grand and Urban Challenge, ${ }^{1-5}$ have demonstrated that laser scanners are versatile sensors able for modern ADAS applications. ${ }^{6-8}$

LIDAR applications for road environments cover different requirements and are not limited to road safety applications. LIDAR may also be used to map the surrounding area and are also useful for localization applications (SLAM). ${ }^{9-15}$

Mapping applications can be performed in both 2D and $3 \mathrm{D}$, depending on the sensors and their capabilities. The main problem associated with $3 \mathrm{D}$ reconstruction is the large volume of data required for processing. This leads to laborious tasks that involve high computational cost, especially when the algorithms are based on pattern matching classification methods. ${ }^{16,17}$ Occupation grids can cope more efficiently with the aforementioned problems; however, they are free space oriented, resulting in increased difficulties regarding obstacle classification. ${ }^{2-4,18,19}$

The main disadvantages associated with LIDAR sensors are based on the small amount of data provided. Other sources of information are usually required to fulfill the requirements for road applications. Techniques, which are capable of handling more than one sensor, are known as data fusion applications. Fusion schemes are separated according to the abstraction level in which the data are fused. For low-level fusion applications, the raw data are fused, ${ }^{5}$ medium-level data fusion is used in applications where the data are obtained and processed; here, several features are extracted from each sensor separately, these characteristics are combined in upper layers. ${ }^{20,21}$ Finally in high-level fusion, each different sensor has its own classifier that determines the type of each obstacle separately, where a final decision is made using the decisions from all the classifiers. ${ }^{22-25}$

Model-based laser scanner classification uses a model to classify the segments found by the laser scanners. In ref. [26], the authors have used bounding boxes to classify obstacles. Clouds of points have been classified according to predefined constraints between points and have made use of a Kalman Filter to predict their movements. ${ }^{27}$ In refs. [28-31], the 
segments have been classified according to a record of the movement of the different obstacles and their behavior along time. The authors of ref. [32] have integrated the patterns that have been detected during a specific time period and provide an estimation of the real shape of the obstacle, where detection is performed according to the shape and predefined constraints. In ref. [9], the authors have presented results on the improvement of detection and classification using localization algorithms based on pattern matching. Reports of pattern classification according to the morphology and occlusion have been provided in ref. [33]. Here, a voting scheme is used, where the classification is improved over time and an estimation of the classification certainty is provided.

Other classifiers perform classification according to feature vectors. In ref. [34], the authors use reflectivity and the size of the objects to create the feature vector. The authors of ref. [35] use these features to create a voting scheme, where a weight is associated with each feature to classify the obstacles. Other attempts ${ }^{36}$ have used the features to create a probability density function for each class, which is used along with a Bayesian filter to determine the type of obstacle. Features vectors may also be created using size information obtained from the laser detection and video data. ${ }^{21}$

Once the obstacle has been detected, the use of a tracking stage has been observed as an interesting technique to avoid false positives, maintain the detection over a period of time and to improve the accuracy of the system. A Kalman Filter is commonly used, ${ }^{37,38}$ while other tracking schemes have made use of particle filters ${ }^{39}$ where other techniques have been presented in refs. [40-44].

One of the problems related with LIDAR detection and classification is due to occlusion from other obstacles on the road. A Laser Scanner beam is a line of sight ray that can easily be occluded by any obstacle located on the road. This is particularly true for 2D LIDARs, where the information is limited to a single detection plane; thus, occlusion is more critical as a result of the limited amount of information provided. The authors of refs. [33] and [45] have attempted to overcome this problem.

\section{Objectives and Methodology}

The following objectives for this area of investigation have been considered:

- Verification of the feasibility of the two LIDAR sensors LD LRS-1000 and LMS-291 for detection in road applications. This will be carried out along with a comparative analysis of their performance and a study of the different scenarios where they can be implemented. Apart from this analysis on the two LIDAR sensor families, the practicality of the laser scanners for ADAS is to be confirmed.

- A new approach for obstacle classification in road environments using laser scanners has been presented. This new approach is checked by performing several tests, which provide important information on the performance of the system.

These tests have been performed within a closed circuit that has been configured specifically for these tests. Two different platforms have been used, a metallic-gray Peugeot 307 and

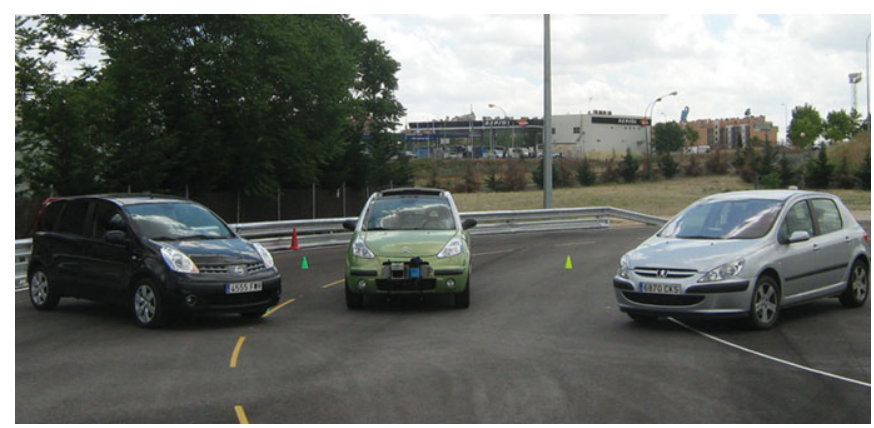

Fig. 1. (Colour online) Test vehicle with radars mounted on the bumper (center), black colored car (left) and gray (silver) colored car (right).

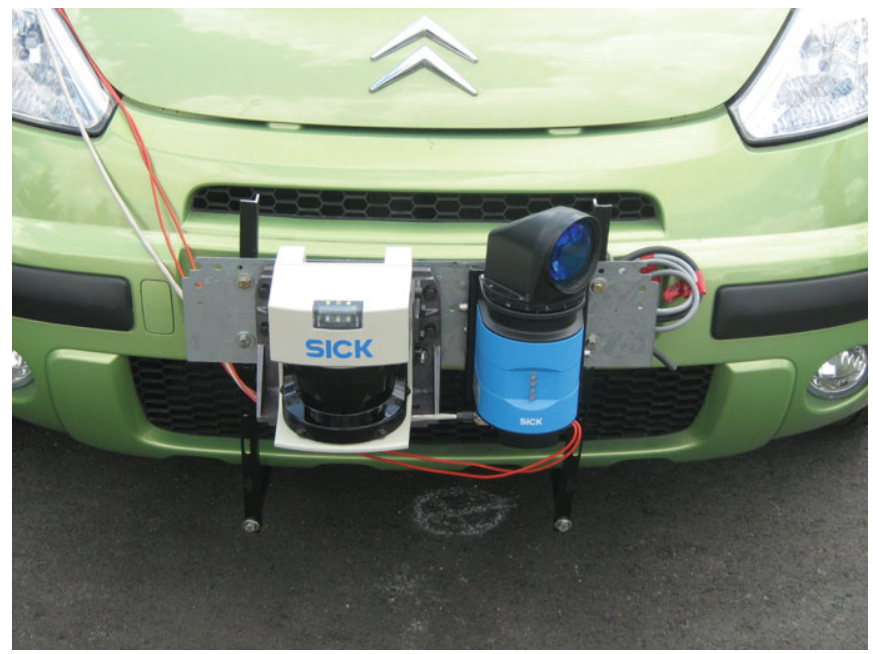

Fig. 2. (Colour online) LMS-291 (left) and LSR 1000 Laser Radar (right).

Table I. A priori comparison of laser performance.

\begin{tabular}{lll}
\hline & LRS-1000 & LMS-291 \\
\hline Field of vision & Up to $360^{\circ}$ & Up to $180^{\circ} / 100^{\circ}$ \\
Angle resolution & $0.125^{\circ}$ to $1.5^{\circ}$ & $0.25^{\circ}$ to $1^{\circ}$ \\
Max distance measure $^{\circ}$ & $250 \mathrm{~m}$ & $80 \mathrm{~m}$ \\
Detection distance $^{a}$ & $229,2 \mathrm{~m}$ & $>80 \mathrm{~m}$ \\
Working frequency $^{\circ}$ & $10 \mathrm{~Hz}$ & $19 \mathrm{~Hz}$
\end{tabular}

${ }^{a}$ There are at least four detection points, from the formula: $d i s t=$ $\frac{d}{2 \operatorname{tg}\left(\frac{\alpha \min }{2}\right)}$, for a car $2 \mathrm{~m}$ width, i.e. $d=0.5 \mathrm{~m}$ (distance between points). It has been considered four points information enough to perform a trustable detection, according to practical results.

a black Nissan Note (Fig. 1). Both of these present the two extreme scenarios found in real applications regarding reflectivity. The metallic-gray vehicle represents the bestcase scenario due to its high reflectivity. The second platform represents the worst-case scenario due its black color. This vehicle presents the most challenging situation for a laser range detector as the amount of reflectivity is lower. Laser scanners have been mounted on the bumper of the cars, as shown in Fig. 2.

\section{LIDAR Comparison}

The sensors that have been used are 2D LIDAR sensors from SICK, each sensor belongs to a different family. A list of their characteristics is presented in Table I. 


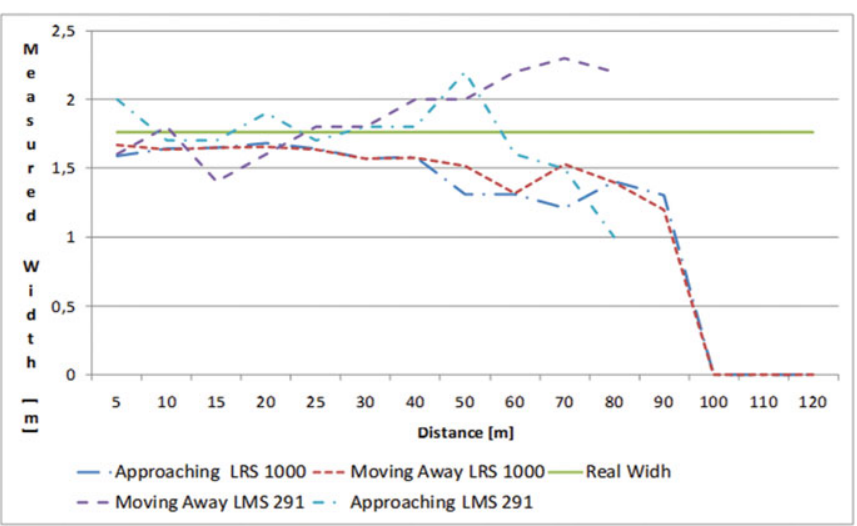

Fig. 3. (Colour online) Width measured vs. distance for metallic vehicle.

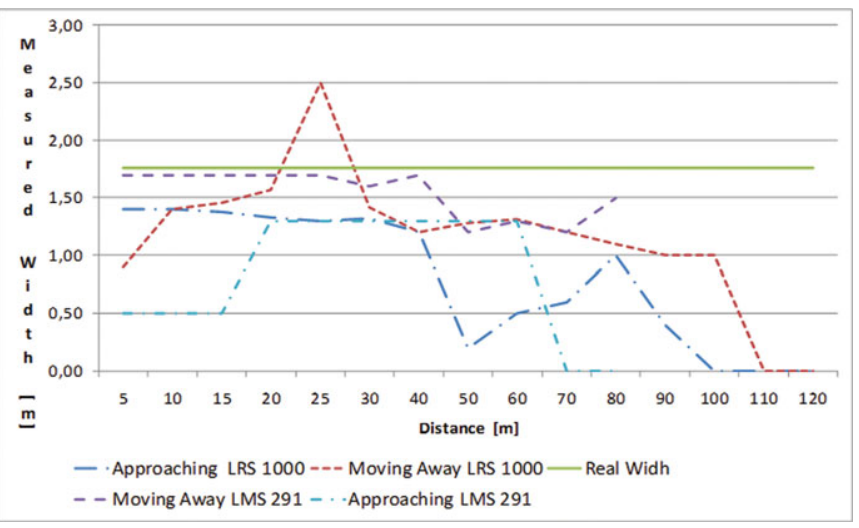

Fig. 4. (Colour online) Width measured vs. distance for black vehicle.

In order to evaluate the accuracy of the measurements, different tests have been performed. All of these have involved approaching and moving away movements.

The resolution selected is the same in both cases $0.25^{\circ}$ as well as the angular resolution for the detection spots.

The index to evaluate the performance of two the lidars is the estimated width of the detected obstacle taking into account that the real widths provided by the manufacturers are

$$
\begin{aligned}
& \text { Nissan note }=1691 \mathrm{~mm}, \\
& \text { Peugeot } 307=1762 \mathrm{~mm} .
\end{aligned}
$$

Width accuracy is very similar for both sensors (Figs. 3 and 4). The measurements from the LMS-291 provide a lower error (Fig. 5). However, the laser radar LRS-1000 provides results for distances greater than $80 \mathrm{~m}$ (Fig. 6).

The LRS-1000 provides higher capabilities that make it a suitable sensor for long-range detections due to the wide range of vision and its exceptionally high resolution. Its lower frequency and large distance measurements show that more structured environments, with less changing conditions and where long distances are important, are typically the best scenarios for this type of LIDAR. Interurban scenarios are a good match for these requirements. For interurban scenarios, detections must be carried out at longer distances $(>200 \mathrm{~m}$ ) due to the speed of the cars involved. In this case, real-time

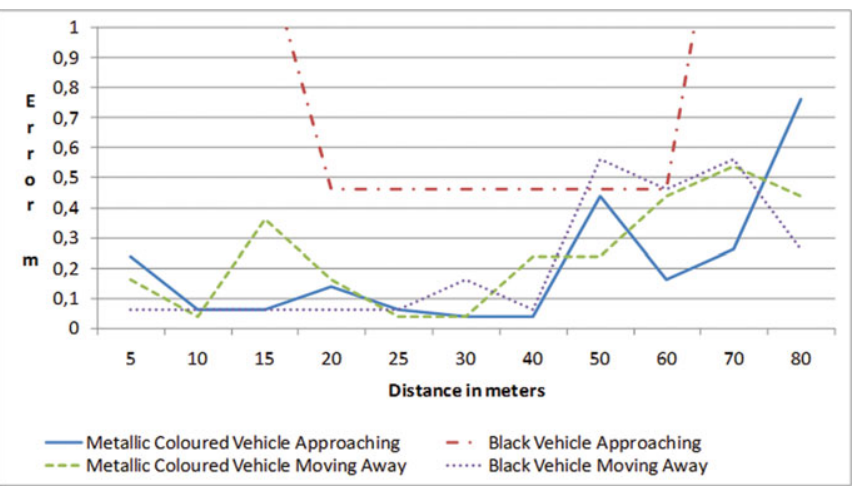

Fig. 5. (Colour online) Error in meters vs. distance for LMS-291 Laser scanner.

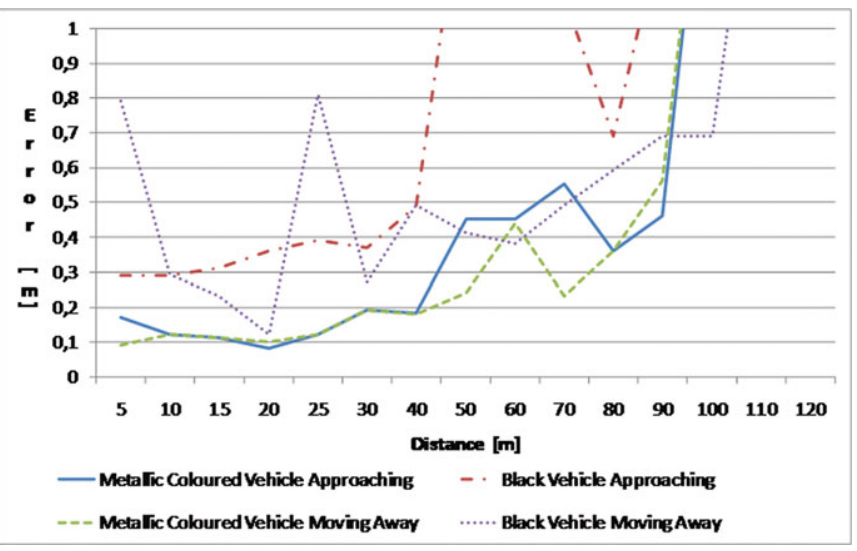

Fig. 6. (Colour online) Error in meters vs. distance for LRS-1000 Laser scanner.

tracking is not of particular importance when compared to the importance of obtaining long-distance detections.

The experiment performed with the LMS-291-S05 provides better results for lower distance ranges. One notable feature of the LMS-291 when compared to the LRS-1000 apart from the lower price is the higher detection frequency. This higher frequency is a very interesting feature when dealing with extreme changing environments, such as urban scenarios; in this case, vehicles have to deal with lower distances and faster changing conditions. In urban scenarios, cars, bikes, pedestrians, and all other types of obstacles may appear from any direction with variable trajectories; in this case, real-time tracking is mandatory to detect dangerous situations in advance while at the same time warning the driver. Thus, in these scenarios, where a fast response is crucial, this sensor is considered as being the best solution.

It also has been proved that detection can be performed under the worst-case scenario, i.e., when the cars to be detected are black. Even though, in some situations, the detection error is high, obstacle detection may still be performed under such severe conditions. Results have also shown that the system is more efficient when detecting obstacles that are moving away as opposed to approaching objects. The reason for this particular behavior is that the front part of the vehicle presents distinctive configurations that hamper the reflection process when compared to the rear of the vehicle. These particular configurations are generally created for aerodynamic proposes to avoid wind forces that increase the amount of power required to move the vehicle. 


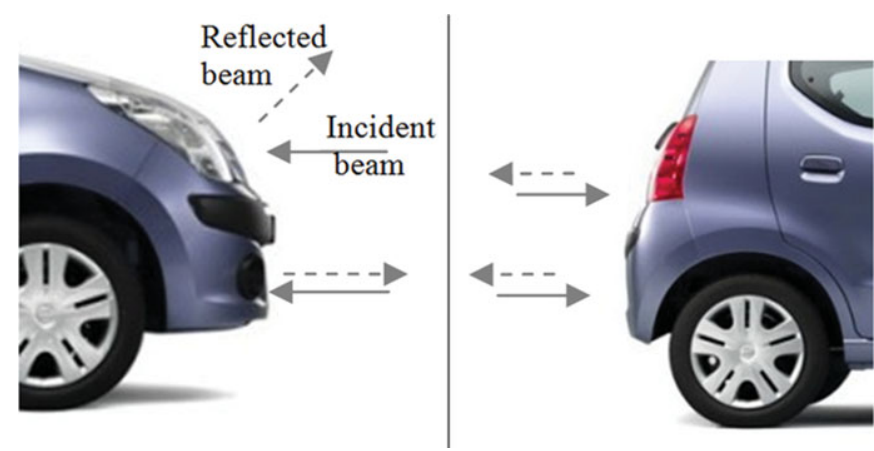

Fig. 7. (Colour online) Comparison of laser reflection for the front and back part of a car. The back of the vehicle has a more structured configuration that aids the detection of laser reflections.

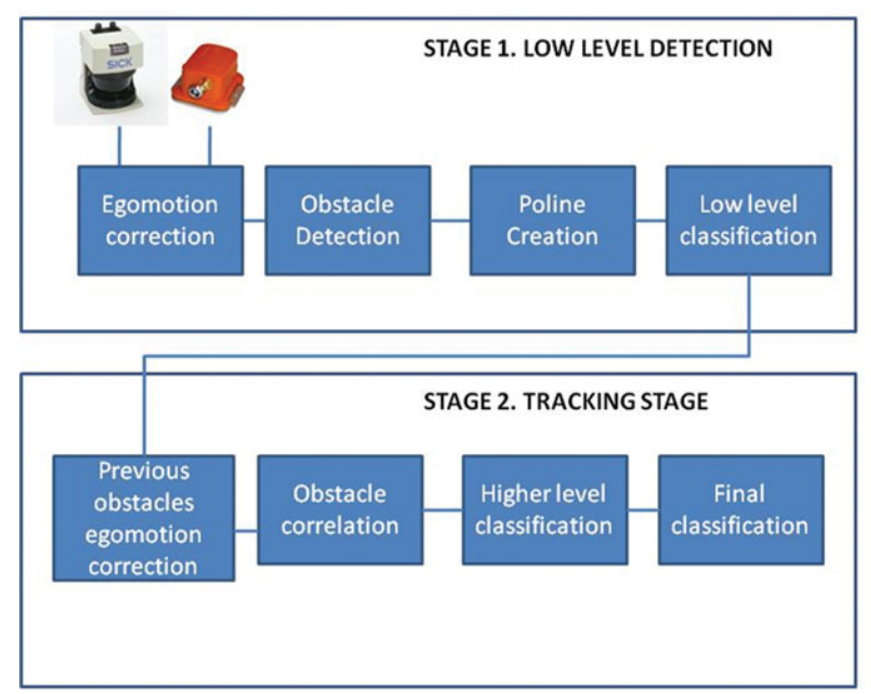

Fig. 8. (Colour online) Processes involved in detection and the classification algorithm.

According to Snell's law, the incident angle and reflected angle are equal with respect to the normal of the surface. According to this law, it is more difficult to detect the front part of the car when compared to the rear, this particular attribute has been verified using this test (Fig. 7).

\section{Road Environment Detection Algorithm}

The main disadvantage with LIDAR is the relatively small amount of information provided even though it is sufficient to provide a first estimation of the shape of the obstacle detected and even to provide some classification results.

Within the scope of this test, an application has been developed to perform low obstacle detection and identification. The algorithm consists of low-level detection and identification, and higher level tracking. This tracking stage not only records and predicts the movement of vehicles and pedestrians, but it is also useful for providing more accurate detections by integrating over the time involved in the process.

The algorithm is composed of two stages (Fig. 8). In the first, the data are received and a low-level identification is performed. In the second stage, the data are integrated over a specific time period, thus a higher classification level is obtained.

\subsection{Stage 1. Low-level detection}

Low-level detection is composed of four systems, where each of them performs a different task that depends on the results from the previous stage.

5.1.1. Egomotion correction. The data received by the laser are corrected according to the movement of the vehicle, to avoid misdetection due to the time difference between the spots, which form part of the scan of the surroundings. Egomotion correction is performed according to the data provided from a GPS sensor included in the system. This GPS Sensor from Xsens (MTI-G) is equipped with egomotion correction and improves the data measured from the GPS system. It provides positioning data and egomotion information, such as velocity, acceleration, and Euler angles (absolute and angular velocity). Velocity and yaw angle measurements are used to compensate the movement of the car for each pulse separately (see Eqs. (1)-(4)):

Translation compensation:

$$
\begin{gathered}
x=x_{0}-v \cdot T_{i} \cdot \cos (\Delta \varphi), \\
y=y_{0}-v \cdot T_{i} \cdot \sin (\Delta \varphi),
\end{gathered}
$$

Rotation compensation:

$$
\begin{gathered}
x=\cos (\Delta \varphi) \cdot x_{0}-\sin (\Delta \varphi) \cdot y_{0}, \\
y=\sin (\Delta \varphi) \cdot x_{0}+\cos (\Delta \varphi) \cdot y_{0},
\end{gathered}
$$

where $v$ is the velocity of the car, $T_{i}$ is the time between the given point and the first point, and $\Delta \varphi$ is the increment in the yaw angle during a period of time $T_{i}$.

5.1.2. Obstacle detection. Once the displacement due to the movement of the car is corrected, the resulting points are joined according to the distance between them by using a clustering algorithm based on the Euclidean distance for a given threshold, which is distance-dependant (Eq. (5)).

$$
t h=t h_{0}+K \cdot d i s t
$$

Here $t h_{0}$ is the threshold base and $K$ is a proportional constant that is multiplied by the distance.

Thus, for a given point $p\left(x_{i}, y_{i}\right)$, it may be treated as belonging to a segment $S_{j}$ if it satisfies

$$
p_{i}\left(x_{i}, y_{i}\right) \in S_{j} \rightarrow\left\{\exists\left[p_{j}\left(x_{j}, y_{j}\right) \in S_{j}\right]: d\left(p_{j}, p_{i}\right)<t h\right\} .
$$

The algorithm checks for all of the points the segments have created and the points among them, if the case arises where a point is not included within any segment, a new segment is then created. After all the points have been verified, the algorithm searches for segments containing only one point, these are then removed as they are considered as false detection points.

In Fig. 9, an example of segment creation is presented. Here, segment A is created, but after the verification process, where only one point is found, this point is removed from the final segments.

5.1.3. Polyline creation. Once the segments are created, the points contained within in each segment are merged using 


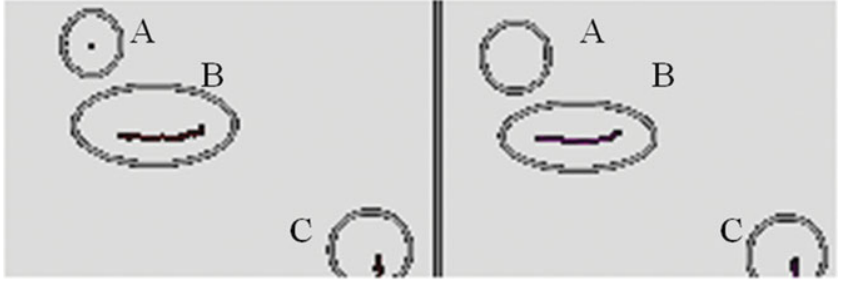

Fig. 9. (Colour online) Segment creation example.
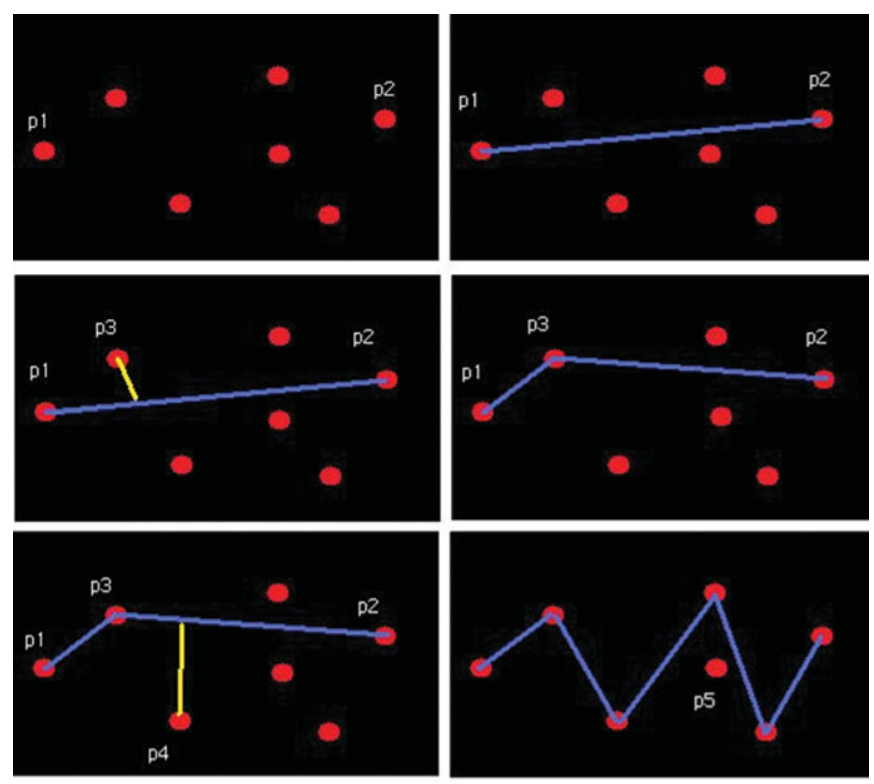

Fig. 10. (Colour online) Example of polyline creation.

lines known as polylines. These lines are merged together according to the distance between the points included in it. This process is a variation of the Ramer algorithm. ${ }^{33}$ The first and last points are merged using a line, for each point contained within this segment, the distance to the line is computed and if it is higher than a given threshold two new lines are created merging these three points. This process is repeated for every point within these new lines.

An example of polyline creation is shown in Fig. 10. As $\operatorname{dis}\left(p_{3}, \operatorname{Rect}\left(p_{1}, p_{2}\right)\right)<t h$, two new lines are created, Rect $\left(p_{1}, p_{3}\right)$ and $\operatorname{Rect}\left(p_{3}, p_{4}\right)$. This process is repeated for $p_{4}$. Finally, the resulting form of the polylines is shown. The Point $p_{5}$ has a smaller distance than the threshold, thus this point is considered to be a spare point.

5.1.4. Low-level classification. Low-level classification is performed with the information provided by the previously described stage. Here, different obstacles can be differentiated

(1) Little obstacles: These are regarded as obstacles, where the size is not compatible with that of a vehicle, buildings or any other large obstacles that are commonly found in the road environment. These obstacles can be considered as regions of interest for further fusion algorithms that use vision to classify the class of obstacles. These are the parts of the road, where important obstacles for road safety can be found, such as pedestrian or bicycles.

(2) L-shaped obstacles: These obstacles are those where the angle and size are similar to a L. In the road environment,

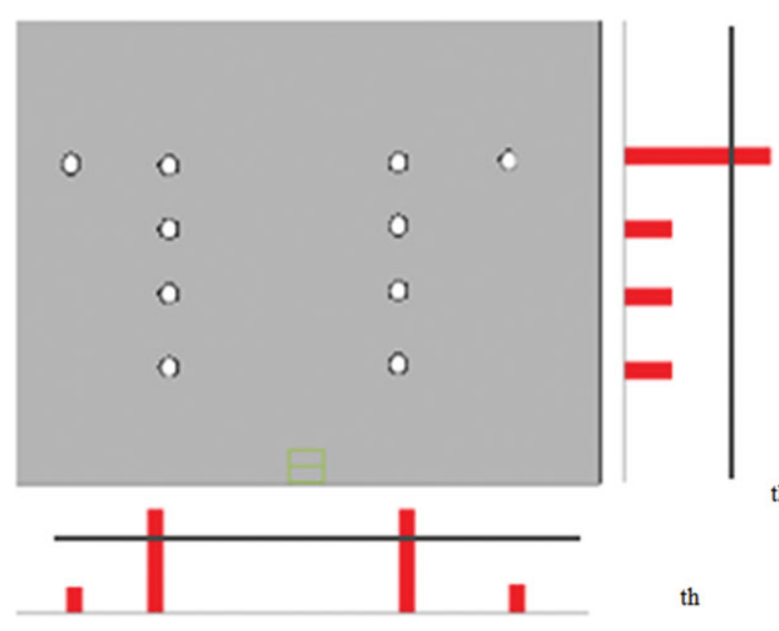

$\mathrm{X}$ axis histogram

Fig. 11. (Colour online) Road border detection using little obstacle histograms.

these are typically buildings or cars. This information can be used for further classifications, which use either fusion algorithms or higher level laser-scanner-based classification algorithms. The classification algorithm checks angles of consecutive lines and looks for angles close to $90^{\circ}$ that represent the L-shaped patterns.

(3) Road limits: Two different possibilities exist for these obstacles. These two detections are performed according to the following procedures:

(a) Big obstacles: If an obstacle bigger than a given threshold $t h$ is found, it is considered as being a candidate for a road limit. The position is checked and if it is located parallel to the trajectory of the car, it is finally labeled as a road limit.

(b) Other road limits detection: At the first stage, only a small amount of little obstacles is found and labeled. After this first classification, two little obstacle histograms that represent the frequency of little obstacles along the $x$ - and $y$-axis of the road are created. If the frequency for a given axis is sufficient, it may be considered that the obstacles found on the road borders can be considered as road limits. If a curve is detected using yaw angle measurements, this detection is disregarded, as road limits are not parallel or perpendicular to the movement of the car. This method although straightforward is demonstrated as being fast and reliable for road border obstacle detection in the majority of scenarios (Fig. 11).

(4) Possible vehicles: The pattern provided by moving obstacles can be differentiated and used to perform vehicle obstacle classification and tracking. These obstacles can be detected using LIDAR LMS-291. For a $0.25^{\circ}$ resolution, it performs four independent scans that provide four sets of spots with a $1^{\circ}$ resolution. Each scan is separated $0.25^{\circ}$ with respect to the previous one (Fig. 12). Thus, after four scans, the LIDAR returns a complete set of spots separated by $0.25^{\circ}$. When a moving obstacle is found, the four scans performed by the LIDAR for a single detection appear with a variation that is proportional to the speed and direction of the detected object and the test vehicle (Fig. 13).

Once this particular pattern with a serrated shape is found, the velocity of the vehicle can be calculated by measuring 

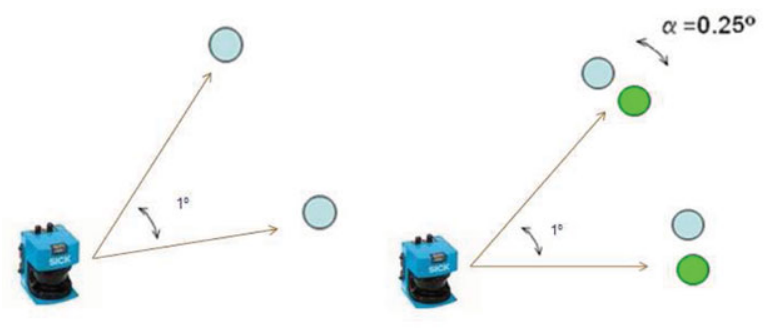

$1^{\text {st }}$ Scan (after $\left.13 \mathrm{msec}\right)$
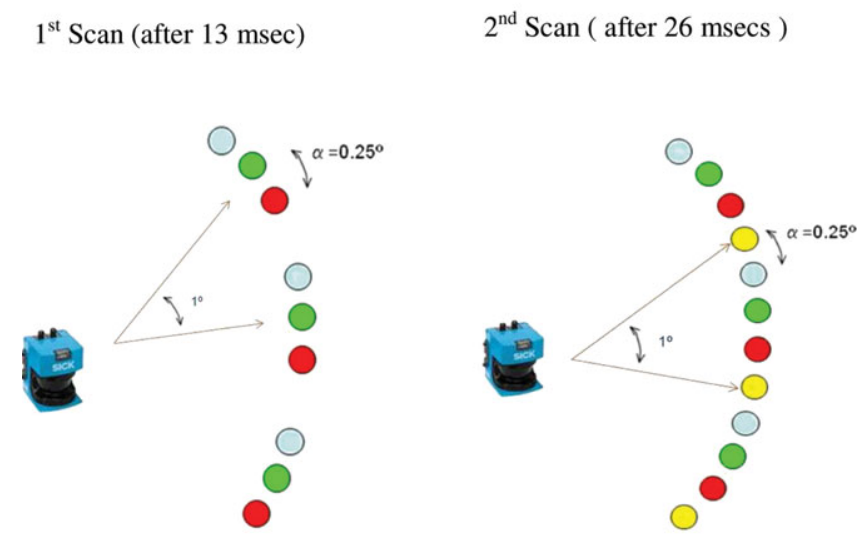

$3^{\text {rd }}$ Scan (after 39 msecs)

$4^{\text {th }}$ Scan (after 52 msecs)

Fig. 12. (Colour online) Laser behavior for a single spot.

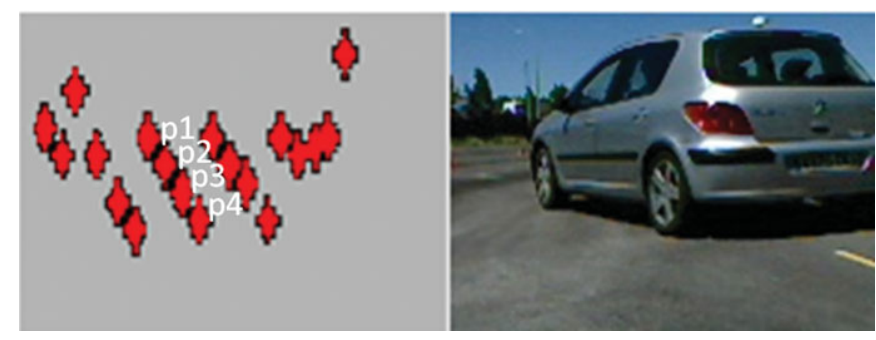

Fig. 13. (Colour online) Moving vehicle pattern.

the distance between two consecutive points (Fig. 12) (see Eqs. (7)-(10))

$$
v=\frac{\sqrt[2]{\left(x_{1}-x_{2}\right)^{2}+\left(y_{1}-y_{2}\right)^{2}}}{T}
$$

Here, $T$ is the rotation period, which is $T=13 \mathrm{~ms}$. Since there are four scans, three different speeds can be measured in order to provide a more reliable measurement

$$
\begin{aligned}
V y & =\frac{\sum_{n=N}^{N-2} \frac{y_{n}-y_{n-1}}{t}}{3}, \\
V x & =\frac{\sum_{n=N}^{N-2} \frac{x_{n}-x_{n-1}}{t}}{3}, \\
v & =\sqrt{v_{x}^{2}+v_{y}^{2}},
\end{aligned}
$$

where $t=13$ msegs and $v$ is in $\mathrm{m} / \mathrm{s}$.

False positives can be avoided by detecting impossible speeds or movements. LRS information cannot give this pattern for a single spot detection, but by combining more than one scan a similar pattern may be detected.

(5) Other Obstacles: These obstacles are those that does not fix with any other patterns previously presented.

\subsection{Stage 2. Higher level classification}

A higher level stage is required to observe the behavior of different obstacles during a specific time period. Previously, scanned obstacles are stored and verified using the new lowlevel detection.

5.2.1. Previous obstacle egomotion correction. The egomotion correction is performed according to the movement of the car in the same way as the low-level detection, explained in the previous section (see Eqs. (1)(4)). Thanks to this correction, all the previously detected obstacles can be referenced to the current vehicle position.

For obstacles labeled as possible vehicles, the velocity of the detected vehicle, according to previous scans, is computed and the next position is calculated taking the movement of the car into account. If there is not enough information available for this tracking process, the low-level speed detected from the previous scan is used to calculate this position. Once the vehicle has been detected during several scans, this velocity is corrected using high-level tracking. This high-level velocity information is considered to be more accurate because it eliminates the laser rotation displacement, which leads to possible measurement errors.

Once the egomotion has been corrected and the movement of possible vehicles is computed, obstacles are searched for within a window according to the size of the obstacles from previous scans. If an obstacle is found within this window of the current scan, a comparison algorithm is used to verify if the obstacle is the same and if several obstacles have been found. The obstacle with the most amount of similarities according to several parameters is considered as being the same obstacle.

5.2.2. Obstacle comparison. The Comparison process is carried out according to shape characteristics, i.e., the width and position (see Fig. 14 and Eqs. (11)-(13)). If all of the comparisons remain within certain values, they are considered as being the same obstacle. If the case arises, where there are several possible candidates, the one with the closest value is considered as being the same obstacle (see Eq. (14))

$$
\begin{gathered}
x_{\text {med }}=x_{\min }+\left(x_{\max }-x_{\min }\right) / 2, \\
y_{\min }, \\
\text { width }=x_{\max }-x_{\min } .
\end{gathered}
$$

These three parameters are the most representative since $Y_{\max }$ is a parameter with excess variation from the point of view of changes or occlusions, thus it has not been considered for comparison purposes

$$
\operatorname{Corr}=\gamma_{1} \cdot d_{\text {width }}+\gamma_{2} \cdot d_{x \text { med }}+\gamma_{3} \cdot d_{y \min } .
$$

Here, $\gamma_{1}$ is the threshold applied to each distance of the different parameters that have been considered. 


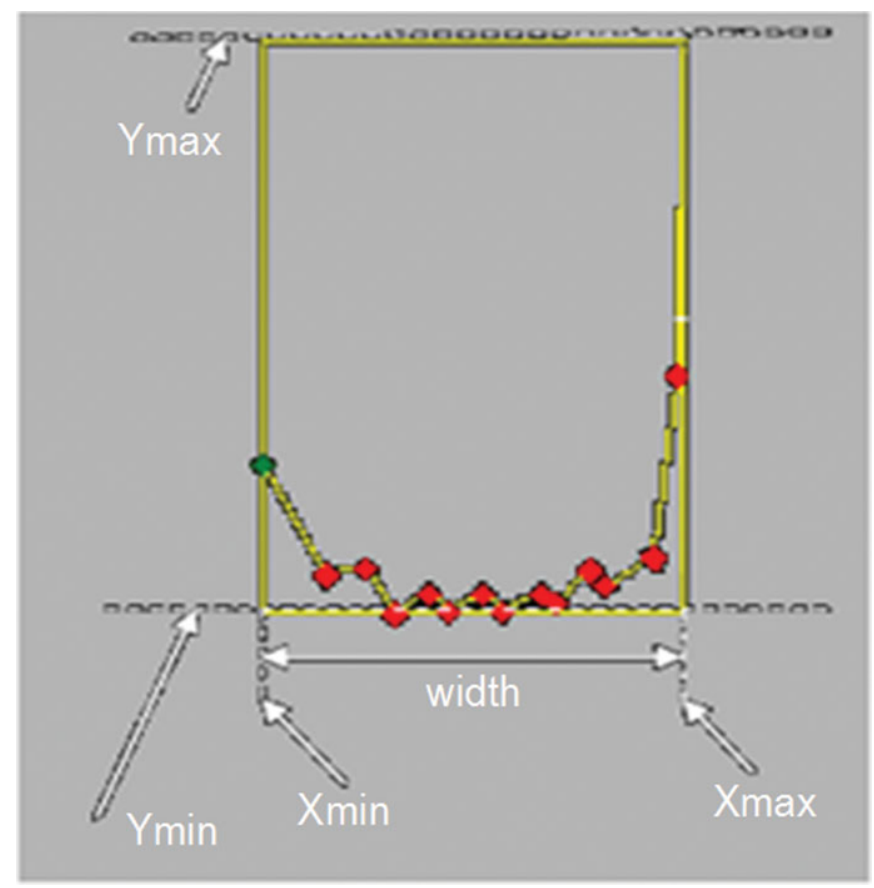

Fig. 14. (Colour online) Shape characteristics for an obstacle.

5.2.3. Higher level classification. The higher level classification algorithm is based on a voting scheme that uses the ten last movements and low-level classification to perform the final decision

$$
V_{i}=\delta_{i} N_{i}
$$

Here, $V_{i}$ represents the number of votes for each type of obstacle, $\delta_{i}$ is a gain factor associated with each obstacle, and $N_{i}$ is the number of times that an obstacle has been considered as being this type during the low-level detection. The biggest $V_{i}$ value represents the type of obstacle that has been detected. Owing to this scheme, a certainty value is provided for the given type of obstacle

$$
p=100 \cdot \frac{V_{i}}{\left(10 \cdot \delta_{i}\right)} .
$$

According to the $p$ formula (see Eq. (16)), for values where the occurrence is 10 , the certainly $(p)$ will be $100 \%$.

5.2.4. Final classification. Before the final classification is carried out, several higher level filters are used to correct possible false positives in the case of possible vehicle detection. These false detections can be avoided by computing the last ten movements stored.

Several filters have been added to avoid false detections. For example, $100 \mathrm{~km} / \mathrm{h}$ is a speed limit for urban scenarios. Also, high lateral movements are considered false detections.

Pitch movements can be detected and the measurement discarded for the majority of incidences thanks to the MTI-G sensor used. This sensor is capable of providing the pitch angle, thus making it possible to detect large changes in the pitch angle, which degrades the quality of the laser scanner detection.
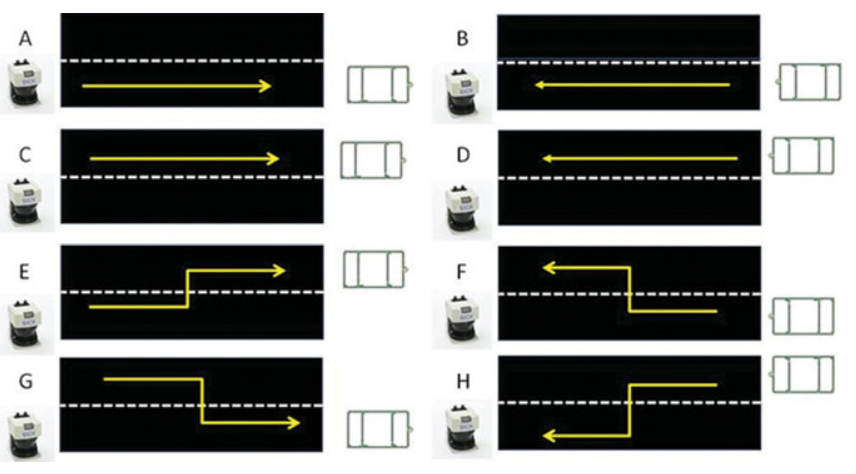

Fig. 15. (Colour online) Test Performed to check the algorithm.

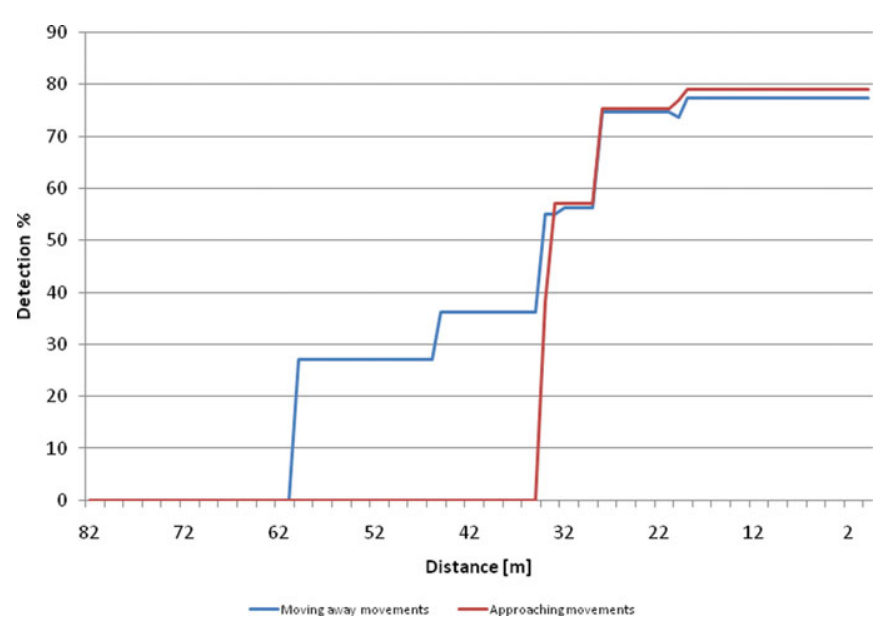

Fig. 16. (Colour online) Detection Percentage vs. distance in meters. Overall results.

\subsection{Test performed}

Several experiments have been performed (Fig. 15) to test the proposed method and verify its feasibility for detecting vehicles. Several of the results from the tests performed have been presented in Fig. 16. Here, the percentage of positive vehicle detections according to the distance is presented. Two kinds of movements have been tested, a vehicle moving toward the LIDAR (approaching movement) and in the opposite direction (moving away movement).

It has been shown in the first part of the present work that the probability of being detected is higher when the car is moving away, i.e., due to the structure of the car. As a result, the detection and classification results are more efficient as a result of the greater amount of information available for this particular situation.

The results presented in Fig. 16 have been obtained when only stage 1 has been used, i.e., only low-level detection. Figure 17 presents results for the complete system. The results have shown that vehicles can be detected within $30 / 40 \mathrm{~m}$ for approaching movements and as high as $80 \mathrm{~m}$ when the object is moving away, this analysis has proved that the complete algorithm is a reliable method and is capable of tracking the movement of cars over short distances. Given the frequency of the LIDAR sensor used (LMS-291) of $19 \mathrm{~Hz}$ and the detection ratios provided in this test, the application presented has proved to be very accurate. As has been previously discussed, in urban environments, the short distances and fast movements are 


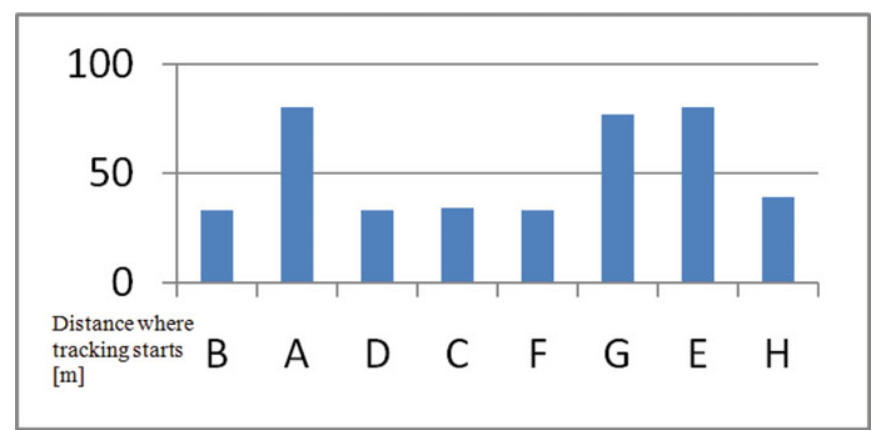

Fig. 17. (Colour online) Tracking distance in meters.

common observed for lower velocities, when compared to interurban environments. These situations are where applications, such as that described in this paper, can improve road safety.

\section{Conclusion and Future Works}

The first conclusion that may be made from the work performed and presented in this paper is that the LIDAR, LMS-291, provides sufficient detection performance, providing a good estimation of the surrounding road environment. However, this technique is ineffective for longrange detections, as it is unable to detect objects located beyond $80 \mathrm{~m}$. The low cost and high frequency of this particular system makes it an interesting option for urban scenarios.

The LIDAR LRS-1000 is a suitable and efficient sensor for road environments, particularly when considering interurban areas, where speeds are generally higher and the detections must be performed for a wide range of distances. In urban areas, both sensors are capable of providing enough information for shape estimation and movement detection; however, the acquisition frequency should also be considered.

It also has been demonstrated that the two LIDARS studied in this paper provide suitable obstacle detection and classification of the vehicles surroundings for real road situations, where the reliability of the vehicle detection is within $30 / 40 \mathrm{~m}$ for approaching objects and up to $80 \mathrm{~m}$ for objects moving away.

The algorithm is based on the operation principle of the LMS-291. This can also be used with the LRS-1000 by integrating several scans and by searching for the pattern variation as is done with the LMS-291.

Test showed that pitch movements could alter the results given by the laser scanner. Thus, a pitching avoiding algorithm was developed. This algorithm detects high pitching movements using the MTI-G GPS sensor, which has accelerometers integrated, able to measure Euler angles. By checking these measurements, high pitching angle changes are detected. Data given by laser scanner are discarded if the case arises that a high pitching movement is detected.

It also has been proved that this system is able to work in real-time conditions, which makes it a suitable application for future ADAS systems. Different weather condition where tested to check the robustness of the algorithm under high stressful conditions.
Future works will focus on data fusion architectures, able to deal with the information from different sensors, providing a more complex set of data. Some of these sensors are laser scanners, computer vision systems, etc. With the use of several sensors, difficulties inherent to each independent sensor can be overcome, providing a complete and trustable data acquisition system able to fulfill the requirements of ADAS applications.

\section{Acknowledgments}

The work reported in this paper has been partly funded by the Spanish Ministry of Science and Innovation (TRA200767786-C02-01, TRA2007-67786-C02-02, and TRA200907505) and the CAM project SEGVAUTO-II.

\section{References}

1. http://www.darpa.mil/grandchallenge/

2. M. Montemerlo, J. Becker, S. Bhat, H. Dahlkamp, D. Dolgov, S. Ettinger, D. Haehnel, T. Hilden, G. Hoffmann, B. Huhnke, D. Johnston, S. Klumpp, D. Langer, A. Levandowski, J. Levinson, J. Marcil, D. Orenstein, J. Paefgen, I. Penny, A. Petrovskaya, M. Pflueger, G. Stanek, D. Stavens, A. Vogt and S. Thrun, "Junior: The stanford entry in the urban challenge," J. Field Robot. Ser. 9, 25, 569 - 597 (Sep. 2008).

3. C. Urmson et al., "Autonomous driving in urban environments: Boss and the urban challenge," J. Field Robot. 25(8), 425-466 (2008).

4. S. Kammel, J. Ziegler, B. Pitzer, M. Werling, T. Gindele, D. Jagzent, J. Schröder, M. Thuy, M. Goebl, F. von Hundelshausen, O. Pink, C. Frese and C. Stiller, "Team AnnieWAY's autonomous system for the 2007 DARPA urban challenge," J. Field Robot. Ser. 9, 25, 615-639 (2008).

5. A. Broggi, A. Cappalunga, C. Caraffi, S. Cattani, S. Ghidoni, P. Grisleri, P. P. Porta, M. Posterli, P. Zani and J. Beck, "The Passive Sensing Suite of the TerraMax Autonomous Vehicle," Proceedings of the IEEE Intelligent Vehicles Symposium 2008, Eindhoven, Netherlands (Jun. 2008), pp. 769-774.

6. Bu. Fanping and C.-Y. Chan, "Pedestrian Detection in Transit Bus Application: Sensing Technologies and Safety Solution," Proceedings of the IEEE International Conference on Intelligent Vehicles (IV '05), Las Vegas, Nevada, USA (2005) pp. 100-105.

7. J. Langheim, A. Buchanan, U. Lages and M. Wahl, "CARSENSE-New Environment Sensing for Advanced Driver Assistance Systems," Proceedings of the International Conference on Intelligent Transportation Systems (ITSC '01), Torino, Italy (Apr. 2001) vol. 3, no. 2, pp. 796-801.

8. D. M Gavrila, M. Kunert and U. Lages, "A Multi-Sensor Approach for the Protection of Vulnerable Traffic Participants the PROTECTOR Project," Proceedings of the 18th IEEE Instrumentation and Measurement Technology Conference (IMTC '01), Budapest, Hungary, (2001) vol. 3, pp. 20442048.

9. C. Wang, C. Thorpe and S. Thrun, "Online Simultaneous Localization and Mapping with Detection and Tracking of Moving Objects: Theory and Results from a Ground Vehicle in Crowded Urban Areas," Proceedings of the IEEE International Conference on Robotics and Automation, Taipei, Taiwan (2003) pp. 842-849.

10. P. Moutarlier and R. Chatila, "Stochastic Mutli-Sensor Data Fusion for Mobile Robot Location and Environment Modeling," Proceedings of the 5th International Symposium on Robotic Research (1989) pp. 207-216.

11. F. Nashashibi and M. Devy, "3D Incremental Modeling and Robot Localization using Laser Range Finder," Proceedings of the IEEE International Conference Robotics and Automation (ICRA'93), Atlanta (May 2-7, 1993) pp. 20-27. 
12. C. Früh and A. Zakhor, "Fast 3D Model Generation in Urban Environment," Proceedings of the International Conference on Multisensor Fusion and Integration of Intelligent Systems, Baden-Baden, Germany (2001) pp. 165-170.

13. H. Zao and R. Shibasaki, "A vehicle-borne urban 3D acquisition system using single-row laser range scanner," IEEE Trans. Syst., Man and Cybern. Part B: Cybern. 33(4), 658-666 (2003).

14. C.-C. Wang, C. Thorpe and A. Suppe, "Ladar-Based Detection and Tracking of Moving Objects from a Ground Vehicle at High Speeds," Proceedings of the IEEE Intelligent Vehicles Symposium (IV'03) (Jun. 2003) pp. 416-421.

15. C.-C. Wang and C. Thorpe, "A Hierarchical Object Based Representation for Simultaneous Localization and Mapping," Proceedings of the IEEE/RSJ International Conference on Intelligent Robots and Systems (IROS '04) (Sep. 28-Oct. 2, 2004) vol. 1, pp. 412-418.

16. T. Rabbani and F. van den Heuvel, "Efficient Hough Transform for Automatic Detection of Cylinders in Point Clouds," Proceedings of the 11th Annual Conference of the Advanced School for Computing and Imaging (ASCI '05), Het Heijderbos, Heijen, The Netherlands (Jun. 2005) pp. 6065.

17. S. J. Ahn, I. Effenberger, S. Roth-Koch and E. Westkämper, "Geometric Segmentation and Object Recognition in Unordered and Incomplete Point Cloud," Proceedings of the DAGM-Symposium (2003) pp. 450-457.

18. S. Thrun, "Learning occupancy grid maps with forward sensor models," Auton. Robots 15(2), 111-127 (2003).

19. M. Tay, "An efficient formulation of the Bayesian occupation filter for target tracking in dynamic environments," Int. J. Veh. Auton. Syst. 6(17), 155-171 (Dec. 31, 2007).

20. U. Hofmann, A. Rieder and E. D. Dickmanns, "Radar and Vision Data Fusion for Hybrid Adaptive Cruise Control on Highways," Proceedings of the International Conference Computing Visual Systems, Vancouver, BC, Canada (2001) pp. 125-138.

21. N. Kaempchen, M. Buehler and K. Dietmayer, "Feature-Level Fusion for Free-Form Object Tracking using Laserscanner and Video," Proceedings of the IEEE Intelligent Vehicles Symposium (Jun. 6-8, 2005) pp. 453-458.

22. A. Broggi, P. Cerri, S. Ghidoni P. Grisleri and H. G. Jung, "Localization and Analysis of Critical Areas in Urban Scenarios," Proceedings of the IEEE Intelligent Vehicles Symposium, Eindhoven, The Netherland (Jun. 4-6, 2008) pp. 1074-1079.

23. C. Premebida, G. Monteiro, U. Nunes and P. Peixoto, "A Lidar and Vision-Based Approach for Pedestrian and Vehicle Detection and Tracking," Proceedings of the IEEE International Conference on Intelligent Transportation Systems, Seattle, WA, USA (Sep.-Oct. 2007) pp. 10441049.

24. J. P. Hwang, S. E. Cho, K. J. Ryu, S. Park and E. Kim, "Multi-Classifier Based LIDAR and Camera Fusion," Proceedings of the IEEE International Conference on Intelligent Transportation Systems, Seattle, WA, USA (Sep.Oct. 2007) pp. 467-472.

25. S. Milch and M. Behrens, "Pedestrian Detection with Radar and Computer Vision," Proceedings of the Conference on Progress in Automobile Lighting, Darmstadt, Germany (2001). http://citeseerx.ist.psu.edu/viewdoc/summary?doi=10.1.1.20.9264

26. H. Zhao, X. W. Shao, K. Katabira and R. Shibasaki, "Joint Tracking and Classification of Moving Objects at Intersection using a Single-Row Laser Range Scanner," Proceedings of the IEEE Intelligent Transportation Systems Conference (ITSC '06), Canada (Sep. 17-20, 2006) pp. 287-294.

27. J. Sparbert, K. Dietmayer and D. Streller. "Lane Detection and Street Type Classification using Laser Range Images," Proceedings of the IEEE Intelligent Transportation Systems
Conference, Oakland, CA, USA (Aug. 25-29, 2001) pp. 454459.

28. K. Fuerstenberg, K. Dietmayer and V. Willhoeft, "Pedestrian Recognition in Urban Traffic Using a Vehicle Based Multilayer Laserscanner," Proceedings of the Intelligent Vehicle Symposium (2002) pp. 31-35.

29. D. Streller, K. Furstenberg and K. Dietmayer, "Vehicle and Object Models for Robust Tracking in Traffic Scenes Using Laser Range Images," Proceedings of the IEEE Intelligent Transportation Systems (2002) pp. 118-123.

30. K. Ch. Fuerstenberg, K. C. J. Dietmayer, S. Eisenlauer and V. Willhoeft, "Multilayer Laserscanner for robust Object Tracking and Classification in Urban Traffic Scenes," Proceedings of the 9th World Congress on Intelligent Transport Systems (ITS '02), Chicago, (Oct. 2002) Paper 2054. pp. 7-8.

31. K. Ch. Fuerstenberg, J. Hipp and A. Liebram "A Laserscanner for Detailed Traffic Data Collection and Traffic Control," Proceedings of the 7th World Congress on Intelligent Transport Systems (ITS '00), Turin, (2000) Paper 2335.

32. G. Gate and F. Nashashibi, "Using Targets Appearance to Improve Pedestrian Classification with a Laser Scanner," Proceedings of the IEEE Intelligent Vehicles Symposium (Jun. 2008) pp. 571-576.

33. F. Nashashibi and A. Bargeton, "Laser-Based Vehicles Tracking and Classification using Occlusion Reasoning and Confidence Estimation," Proceedings of the IEEE Intelligent Vehicles Symposium (Jun. 2008) pp. 847-852.

34. T. Ogawa and K. Takagi, "Lane Recognition Using On-Vehicle LIDAR," Proceedings of the IEEE Intelligent Vehicles Symposium (2008) pp. 540-545.

35. A. Mendes, L.C. Bento and U. Nunes, "Multi-Target Detection and Tracking with a Laser Scanner," Proceedings of the IEEE Intelligent Vehicles Symposium (Jun. 14-17, 2004) pp. 796-801.

36. C. Premebida and U. Nunes: "A Multi-Target Tracking and GMM Classifier for Intelligent Vehicles," Proceedings of the IEEE Intelligent Transportation Systems Conference, Canada (2006) pp. 313-318.

37. R. A. MacLachlan and C. Mertz, "Tracking of Moving Objects from a Moving Vehicle Using a Scanning Laser Rangefinder," Proceedings of the IEEE Intelligent Transportation Systems Conference (ITSC '06) (Sep. 17-20, 2006) pp. 301306.

38. A. Fod, A. Howard and M Mataric, "A Laser-Based People Tracker," Proceedings of the IEEE International Conference on Robotics and Automation (2002) pp. 3024-3029.

39. D. Schulz, W. Burgard, D. Fox and A. B. Cremers, "Tracking Multiple Moving Targets with a Mobile Robot Using Particle Filters and Statistical Data Association," Proceedings of the IEEE International Conference on Robotics and Automation (2001) vol. 2, pp. 1665-1670.

40. Y. Bar-Shalom and T. E. Fortmann, "Tracking and Data Association (Mathematics in Science and Engineering) (Academic Press, San Diego, CA, USA, 1988).

41. Y. Bar-Shalom, "Tracking methods in a multitarget environment," IEEE Trans. Autom. Control, 23(4), (Aug. 1978).

42. Y. Bar-Shalom and X.-R. Li, Multitarget-Multisensor Tracking: Principles and Techniques (Yaakov Bar-Shalom, Danvers, MA, USA, 1995).

43. S. Blackman and R. Popoli, Design and Analysis of Modern Tracking Systems (Artech House, MA, USA, 1999).

44. D. B. Reid, "An algorithm for tracking multiple targets," IEEE Trans. Autom. Control, 24(6), 843-854 (Dec. 1979).

45. F. García, P. Cerri, A. Broggi, J. M. Amingol and A. de la Escalera, "Vehicle Detection Based on Laser Radar" Proceedings of the European Conference on Computer Aided Systems Theory (EUROCAST '09) (R. Moreno-Diaz, F. Pichler and A. Quesada-Arencibia, eds.). Lecture Notes In Computer Science, Vol. 5717. (Springer-Verlag, Berlin, Heidelberg, 2009) pp. 391-397. 